\title{
Regioselective synthesis of 2-(bromomethyl)-5-aryl-thiophene derivatives via palladium (0) catalyzed suzuki cross-coupling reactions: as antithrombotic and haemolytically active molecules
}

\author{
Komal Rizwan ${ }^{1}$, Muhammad Zubair ${ }^{1 *}$, Nasir Rasool ${ }^{1 *}$, Shaukat Ali ${ }^{1}$, Ameer Fawad Zahoor ${ }^{1}$, Usman Ali Rana ${ }^{2}$, \\ Salah Ud-Din Khan², Muhammad Shahid ${ }^{3}$, Muhammad Zia-UI-Haq ${ }^{4}$ and Hawa ZE Jaafar ${ }^{5 *}$
}

\begin{abstract}
Background: It is seen that the regioselective functionalizations of halogenated heterocycles play an important role in the synthesis of several types of organic compounds. In this domain, the Suzuki-Miyaura reaction has emerged as a convenient way to build carbon-carbon bonds in synthesizing organic compounds. Some of the most important applications of these reactions can be seen in the synthesis of natural products, and in designing targeted pharmaceutical compounds. Herein, we present the regioselective synthesis of the novel series of 2-(bromomethyl)-5-aryl-thiophenes 3a-i, via Suzuki cross-coupling reactions of various aryl boronic acids with 2-bromo-5-(bromomethyl)thiophene (2).

Results: The synthesized compounds were screened for their haemolytic and antithrombolytic activities. The novel compounds 3f, 3i showed highest 69.7, 33.6\% haemolysis of blood cells, respectively. The antithrombolytic activity of the compounds was found to be within low to moderate against human blood clot. The compound $3 \mathbf{i}$ showed potent clot lysis (31.5\%).
\end{abstract}

Conclusions: Considering these results, it is concluded that the synthesized compounds can be used as a promising source of therapeutic agents.

Keywords: Thiophene, Palladium, Suzuki cross-coupling reactions, Heterocycles, Aryl boronic acid, Antithrombotic, Haemolytic, Cytotoxicity

\section{Background}

In present days, the synthesis of new and safe therapeutic agents is getting high importance in the field of medicinal science and pharmaceuticals. Most precisely, sulphur containing heterocycles are seen as the center of activity due to their widespread use in several important medicinal compounds. However, it is seen that the success of thiophene as an important moiety of medicinal

\footnotetext{
*Correspondence: zubairmkn@yahoo.com; nasirhej@yahoo.co.uk;

hawazej@gmail.com

'Department of Chemistry, Government College University, Faisalabad 38000, Pakistan

${ }^{5}$ Department of Crop Science, Faculty of Agriculture, 43400 UPM Serdang, Selangor, Malaysia

Full list of author information is available at the end of the article
}

agents led to the introduction of new therapeutic drugs. Substituted thiophene derivatives are well known for their chemotherapeutic applications. Many thiophene based heterocyclic compounds have shown versatile pharmacological activities such as antimicrobial [1,2], antiamoebic [3], antiparasitic, anticancer [4], antifolates, antipsychotic [5], diabetes mellitus [6], anticonvulsant [7], analgesic [8], antidepressant [9], antihistaminic, anticholinergic [10], antiallergic [11]. In addition, the cholesterol inhibition activity and as antagonist against many hormones releasing receptors has also been reported. In a yet different context, the thiophene based heterocyclic compounds has also been employed in formulizing computer printer's ink and as a raw material for herbicides and pesticides [12]. 
Some of the recent studies showed that the thiophene containing compounds constitutes an important class of materials, which show intrinsic electronic properties such as luminescence, redox activity, non-linear optical chromism and electron transport [13-17]. The incidence of death due to thrombosis is higher in the world. Antithrombolytic activity of thiophene based compounds has been reported in literature $[18,19]$.

In the synthesis of several types of organic compounds, the transition metal-catalyzed reactions are well known for the formation of new carbon-carbon $(\mathrm{C}-\mathrm{C})$ bonds. In this context, the Pd-catalyzed Suzuki-Miyaura coupling reaction [20] is one of the most efficient and unique method for the $\mathrm{C}-\mathrm{C}$ bonds formation due to the requirement of mild reaction conditions, easily available environmentally safe organoboron compounds, high tolerance of functional groups and easy handling of the byproducts [21-25].

The Suzuki-Miyaura cross-coupling reaction, which produces biaryls has proven to be the most important building blocks in organic synthesis owing to their industrial applications. We have previously reported the synthesis of arylthiophenes by regioselective Suzuki cross-coupling reactions and they were potentially studied as pharmaceutical agents [26,27].

There are few reports about the Suzuki Cross Coupling reaction of benzyl halides with different palladium catalysts under variable reaction conditions. Langle et al. [28] reported the Suzuki cross coupling reaction of unsymmetrical diarylmethanes, while Bandgar et al. [29] reported ligand free Suzuki cross coupling reactions of benzylic halides with aryl boronic acid. Molander and Elia [30] described the Suzuki-Miyaura cross-coupling Reactions of Benzyl halides with Potassium Aryltrifluoroborates. The cross-coupling of benzylic bromides with various aryl boronic acids have also been reported under microwave conditions [31].

For the first time, the present work focuses on the synthesis of various palladium (0) catalyzed Suzuki cross coupled derivatives of 2-bromo-5-(bromomethyl) thiophene, particularly with the aim to investigate their biological activities (Haemolytic and Antithrombolytic activities).

\section{Results and discussion Chemistry}

We have investigated the Suzuki cross coupling reactions of 2-bromo-5-(bromomethyl)thiophene (2) with various aryl boronic acids under optimized conditions. To the best of our knowledge, no such work on the synthesis and biological activities of 2-(bromomethyl)-5-aryl-thiophenes $(3 \mathbf{a}-\mathbf{i})$ has been reported to date.

As outlined in the reaction scheme (1), the first step in the synthesis of 2-(bromomethyl)-5-aryl-thiophenes (3a-i) is the preparation of intermediate compound 2-bromo-5(bromomethyl)thiophene (2), which was obtained in 58\% yield from the reaction between 2-methylthiophene (1) and $\mathrm{N}$-bromosuccinamide in $\mathrm{CCl}_{4}$ [32] (Scheme 1).

In the next step, the Suzuki cross coupling reaction of appropriate aryl boronic acids with 2-bromo-5-(bromomethyl) thiophene (2) was carried out that eventually led to the corresponding 2-(bromomethyl)-5-aryl-thiophenes (3a-i) in moderate to excellent yields ( $25-76 \%$ ) (Scheme 1, Table 1$)$.

The Suzuki-Miyaura cross coupling reaction of benzyl halide with aryl boronic acid usually follow slow oxidative addition and facile reductive elimination as compared to aryl halide [33,34], therefore the reaction of aryl halide with aryl boronic acid is preferred over benzyl halide. The structures of these newly synthesized compounds were investigated from the data based on elemental analyses, Mass spectrometry, ${ }^{1} \mathrm{H}-\mathrm{NMR}$ and ${ }^{13} \mathrm{C}-\mathrm{NMR}$ spectra.

The results revealed that the compound 2-(bromomethyl)5-(4 methoxyphenyl)thiophene (3b) was obtained in excellent yield (76\%), which could be due to the solvent mixture (1,4-dioxane/water $=4: 1$ ), which has previously been reported to obtain high yields [35]. Another possible explanation is the high solubility of oxygen containing boronic acids in 1,4-dioxane, which led to the obtained high yield of compound (3b). The coupling of intermediate compound (2) with 3-chloro-4-fluoro phenyl boronic acid also gave 2-(bromomethyl)-5-(3-chloro-4-fluorophenyl) thiophene (3a) in good yield. The products 2-(bromomethyl)5-(4-chlorophenyl)thiophene (3c), 2-(bromomethyl)-5(3,5-difluorophenyl)thiophene (3d), 1-(3-(5-(bromomethyl) thiophene-2-yl)phenyl)ethanone (3e), 2-(bromomethyl)5-(3,5-dimethylphenyl)thiophene (3i) were also obtained in relatively high yields $\sim 63,61,63,70 \%$ respectively. The obtained yield of products 2-(bromomethyl)-5-(4 (methylthio)phenyl)thiophene (3f), 2-(bromomethyl)-5-

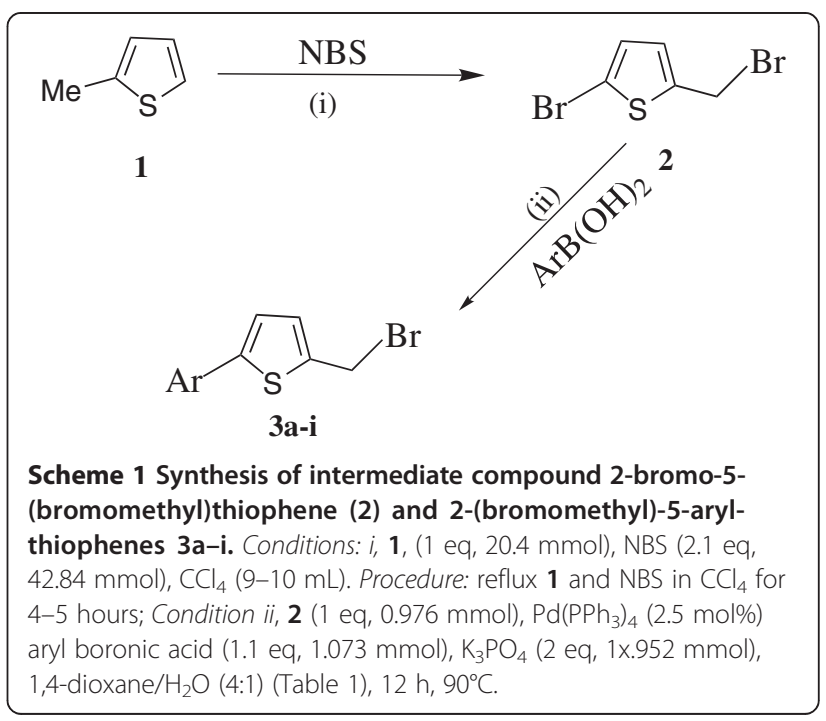


Table 1 Synthesis of 2-(bromomethyl)-5-aryl-thiophenes (3a-i)$$
3
$$

Aryl/ArylBoronic acids

$3-\mathrm{Cl}-4-\mathrm{F}-\mathrm{C}_{6} \mathrm{H}_{3}$

Products

Solvent/ $\mathrm{H}_{2} \mathrm{O}(4: 1)$

1,4-Dioxane

Yield \%<smiles>Fc1ccc(-c2ccc(CBr)s2)cc1Cl</smiles>

b

4-MeO- $\mathrm{C}_{6} \mathrm{H}_{4}$<smiles>COc1ccc(-c2ccc(CBr)s2)cc1</smiles>

1,4-Dioxane

76<smiles>Clc1ccc(-c2ccc(CBr)s2)cc1</smiles>

1,4-Dioxane

C $\quad 4-\mathrm{Cl}_{-} \mathrm{C}_{6} \mathrm{H}_{4}$

d $\quad 3,5-\mathrm{F}_{2}-\mathrm{C}_{6} \mathrm{H}_{3}$<smiles>Fc1cc(F)cc(-c2ccc(CBr)s2)c1</smiles>

1.4-Dioxane

61<smiles>CC(=O)c1cccc(-c2ccc(CBr)s2)c1</smiles>

1,4-Dioxane<smiles>CSc1ccc(-c2ccc(CBr)s2)cc1</smiles>

1,4-Dioxane

g

4-I- $\mathrm{C}_{6} \mathrm{H}_{4}$<smiles>BrCc1ccc(-c2ccc(I)cc2)s1</smiles>

1,4-Dioxane

60

h $\quad 4-\mathrm{Me}^{-\mathrm{C}_{6}} \mathrm{H}_{4}$<smiles>Cc1ccc(-c2ccc(CBr)s2)cc1</smiles>

1,4-Dioxane<smiles>Cc1cc(C)cc(-c2ccc(CBr)s2)c1</smiles>

1,4-Dioxane 70 
(4-iodophenyl)thiophene (3g), 2-(bromomethyl)-5-ptolylthiophene (3h) was fair as well (Table 1). In the cases, where low yield of products were obtained, the steric effects of substituents attached on aryl group of boronic acids and some practical problems associated with difficult chromatographic purification are suggested to be the possible issues [36]. Ortho-substituted aryl boronic acids have lack of reactivity and cannot couple in a good way due to steric factor. Hence, boronate anion of boronic acid are unable to attack easily on the substrate [36].

\section{Biology \\ Measurement of potential cytotoxicity by haemolytic activity}

The cytotoxicity of the synthesized compounds viz. 2 and $3 \mathbf{a}-\mathbf{i}$ was studied by examining the haemolytic activity against human red blood cells. The cytotoxicity of blood lymphocytes, thymocytes and spleen cells of various compounds are already known [37]. When compared with the positive control triton X-100 standard, the novel compounds $\mathbf{3 f}$ and $\mathbf{3 i}$ showed significantly high haemolytic activity 69.7 and $33.6 \%$ lysis of blood cells respectively, which can be attributed to the presence of electron donating methyl groups. Molongi et al. [38] reported that the anticancer activity is often enhanced by the presence of electron releasing groups. In contrast, the compounds viz. 2, 3a, 3b, 3c, 3d, 3e, 3g and $\mathbf{3 h}$ exhibited haemolytic activity below $10 \%$ (Table 2 , Figure 1). In view of the observed differences in the $\%$ lysis of $\mathrm{RBC}$ values, it is inferred that the electron withdrawing and electron donating nature of the substituent groups have an influence on the haemolytic activity of the compounds [39]. Moreover, the cytotoxicity of the compounds viz. $\mathbf{3 f}$ and $3 \mathbf{i}$ can be optimized by making

Table 2 Cytotoxicity studies by Haemolytic activity of synthetic compounds 2 and $3 a-i$

\begin{tabular}{lc}
\hline Compounds & \% of haemolysis \\
\hline $\mathbf{2}$ & $3.06 \pm 0.03$ \\
3a & $1.63 \pm 0.02$ \\
3b & $6.31 \pm 0.07$ \\
3c & $3.88 \pm 0.04$ \\
3d & $4.43 \pm 0.05$ \\
3e & $5.13 \pm 0.05$ \\
3f & $69.7 \pm 1.23$ \\
3 g & $9.87 \pm 0.08$ \\
3 h & $3.59 \pm 0.03$ \\
3i & $33.6 \pm 0.87$ \\
Phosphate-buffered saline (PBS) & $0.00 \pm 0.00$ \\
Triton X-100 & $100 \pm 0.58$ \\
\hline
\end{tabular}

The results are average \pm S.D of triplicate experiments $p<0.05$. appropriate changes in the molecular structures for the purpose of their use as toxic compounds to control the uncontrolled proliferation of cells [38].

\section{Antithrombolytic activity}

In the field of antithrombotic research, the compounds that exhibit anti-aggregatory activity have received a great deal of interest from research community. Several types of drugs such as heparin, ticlopidine, clopidogrel, plasminogen activator ( $\mathrm{t}-\mathrm{PA}$ ), urokinase and streptokinase were explored as clot lysis agent but only few of them were found potent for clinical purposes [40-43].

Because of high death rate due to cardio-vascular diseases, the clot lysis activity is very important characteristic of any drug $[44,45]$. The compounds under investigation exhibited low to moderate antithrombolytic activity against clot development in human blood. The compound $3 \mathbf{i}$ showed potent clot lysis ( 31.5\%), whereas, the compounds $3 \mathbf{e}$ and $\mathbf{2}$ showed comparatively low thrombolytic activity (Table 3, Figure 2). The values of \% clot lysis for other compounds were found moderate. However, the results were significant $p<0.05$, when compared with streptokinase taken as control. According to the best of our knowledge, no relative literature is available on this type of activity for such compound.

\section{Experimental}

\section{General}

The melting points of compounds were determined using a Buchi melting point apparatus (B-540). High pure analytical grade reagents were used throughout all experiments and were purchased from Sigma Aldrich Chemical Co. (St. Louis, MO, USA) and Alfa Aesar Chemical Co. (St. Parkridge Ward Hill, MA, USA). ${ }^{1} \mathrm{H}-\mathrm{NMR}$ and ${ }^{13} \mathrm{C}$-NMR spectra were measured in $\mathrm{CDCl}_{3}$ and $\mathrm{CD}_{3} \mathrm{OD}$ (Bruker Aspect AM-300 instrument) at 300/75 MHz respectively. The chemical shift values $(\delta)$ were given in ppm and coupling constant was measured in Hertz (Hz). EI-MS spectra were recorded on a JMS-HX-110 spectrometer with a data system. Elemental analysis was carried out by using CHNS/O analyzer (Perkin-Elmer 2400 series). For column chromatography, silica gel (70-230 mesh) and silica gel (230-400 mesh) were used. The reactions were monitored on TLC, using Merck silica gel $60 \mathrm{PF}_{254}$ cards. The compounds were visualized by UV lamp (254-365 nm).

\section{General procedure for synthesis of 2-bromo-5- (bromomethyl)thiophene (2)}

To carry out bromination of 2-methylthiophene, a weighed amount of starting material 2-methylthiophene (1, 1 eq, $20.4 \mathrm{mmol}$ ) suspended in $9-10 \mathrm{~mL}$ of dry carbon tetrachloride $\left(\mathrm{CCl}_{4}\right)$ was made to react with $\mathrm{N}$ bromosuccinimide (NBS) (2.1 eq, $42.84 \mathrm{mmol})$. This reaction mixture is then heated under reflux for four to five 


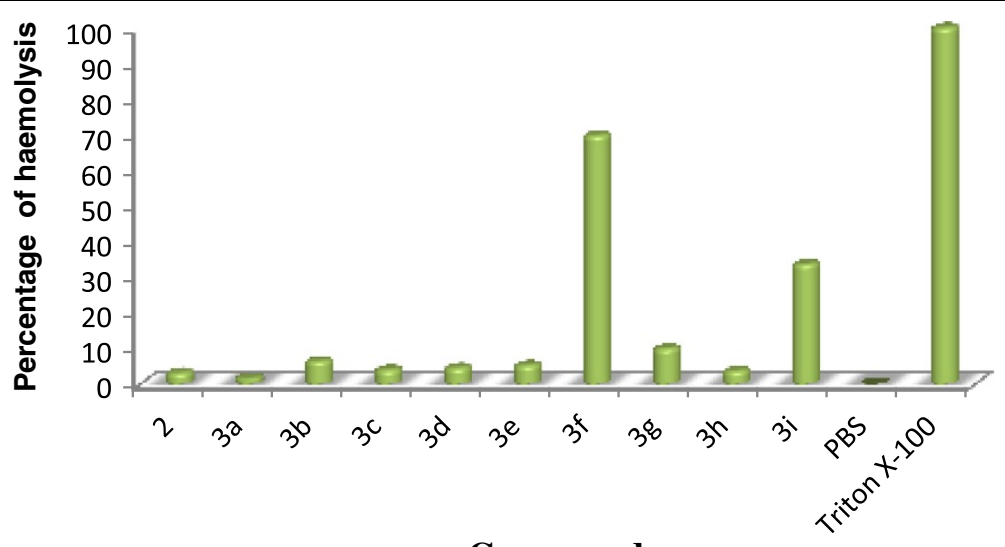

\section{Compounds}

Figure 1 Percentage of haemolysis of synthetic compounds 2, 3a-i.

hours, followed by filtration and the removal of carbon tetrachloride under vacuum. Later on, the synthesized compound was purified by fractional distillation. Finally, the spectroscopic techniques were used to characterized the purified final product [32].

\section{General procedure for the preparation of 2- (bromomethyl)-5-aryl-thiophene (3a-i)}

$\mathrm{Pd}\left(\mathrm{PPh}_{3}\right)_{4}$ (2.5 mol\%) was added to 2-bromo-5-(bromomethyl) thiophene (2, 1 eq, $0.976 \mathrm{mmol})$ under nitrogen atmosphere and the resulting mixture was stirred for $30 \mathrm{~min}$ with the addition of 1,4-dioxane $(2.5 \mathrm{~mL})$. After $30 \mathrm{~min}$ the aryl boronic acid (1.1 eq, $1.073 \mathrm{mmol}$ ), $\mathrm{K}_{3} \mathrm{PO}_{4}(2 \mathrm{eq}$, $1.952 \mathrm{mmol})$ and water $(0.625 \mathrm{~mL})$ were added [27]. The whole mixture was stirred for $12 \mathrm{~h}$ at $90^{\circ} \mathrm{C}$, and was later removed and cooled to room temperature. After cooling to ambient temperature, the mixture was diluted with ethyl acetate and the organic layer was separated, dried with

Table 3 Percentage efficiency of Clot lysis of synthetic compounds 2 and $3 a-i$

\begin{tabular}{ll}
\hline Compounds & Clot lysis \% \\
\hline $\mathbf{2}$ & $2.73 \pm 0.03$ \\
3a & $9.76 \pm 0.08$ \\
3c & $12.3 \pm 0.15$ \\
3b & $5.29 \pm 0.04$ \\
3d & $3.71 \pm 0.04$ \\
3e & $1.96 \pm 0.02$ \\
3f & $8.05 \pm 0.07$ \\
3 g & $7.28 \pm 0.06$ \\
3 h & $4.37 \pm 0.02$ \\
3i & $31.5 \pm 0.45$ \\
Water & $0.43 \pm 0.005$ \\
Streptokinase & $87.2 \pm 0.95$
\end{tabular}

The results are average \pm S.D of triplicate experiments $p<0.05$. magnesium sulfate and the solvent was removed under vacuum. The obtained crude residue was purified by column chromatography using ethyl-acetate and $n$-hexane in (1:1) ratio to get the desired products, which were further analyzed by using different spectroscopic techniques.

\section{Characterization data}

2-bromo-5-(bromomethyl)thiophene (2). Brown liquid; ${ }^{1} \mathrm{H}-\mathrm{NMR}\left(300 \mathrm{MHz}, \mathrm{CDCl}_{3}+\mathrm{CD}_{3} \mathrm{OD}\right): \delta=6.98(\mathrm{~d}, J=$ $3.6 \mathrm{~Hz}, 1 \mathrm{H}), 6.83(\mathrm{~d}, J=3.6 \mathrm{~Hz}, 1 \mathrm{H}), 4.68\left(\mathrm{~s}, 2 \mathrm{H}-\mathrm{CH}_{2}\right)$. ${ }^{13} \mathrm{C}-\mathrm{NMR}\left(75 \mathrm{MHz} \mathrm{CDCl}_{3}+\mathrm{CD}_{3} \mathrm{OD}\right): \delta=25.4,108.2$ 128.0, 128.8, 141.5; EI/MS m/z (\%:) $255.0\left[\mathrm{M}^{+}\right.$] 257.0 $\left[\mathrm{M}^{+}\left(\mathrm{Br}^{79}, \mathrm{Br}^{81}\right)\right.$ 100], $259.0\left[\mathrm{M}^{+}\left(\mathrm{Br}^{81}, \mathrm{Br}^{81}\right) 40\right],\left[\mathrm{M}^{+}-\mathrm{Br}\right]=$ 177.0; $\left[\mathrm{M}^{+}-\mathrm{CH}_{2} \mathrm{Br}\right]=163.0 ;\left[\mathrm{M}^{+}-2 \mathrm{Br}\right]=125.8 ;\left[\mathrm{M}^{+}-\mathrm{Br}\right.$ and $\mathrm{CH}_{2} \mathrm{Br}$ fragment] $=84.0$. Anal. Calcd. for $\mathrm{C}_{5} \mathrm{H}_{4} \mathrm{Br}_{2} \mathrm{~S}$ : C, 23.46; H, 23.50. Found: C, 1.60; H, 1.60\%.

2-(bromomethyl)-5-(3-chloro-4-fluorophenyl)thiophene (3a). Light yellow solid, Mp: 200-250 ${ }^{\circ} \mathrm{C}$; ${ }^{1} \mathrm{H}-\mathrm{NMR}(300 \mathrm{MHz}$, $\left.\mathrm{CDCl}_{3}+\mathrm{CD}_{3} \mathrm{OD}\right): \delta=7.54(\mathrm{~m}, 1 \mathrm{H}$-aryl $), 7.52(\mathrm{~m}, 1 \mathrm{H}$-aryl), 7.36 (s, 1H-thiophene), 7.28 (m, 1H-aryl), 6.77 (s, 1Hthiophene), 4.07 (s, 2H-CH$) .{ }^{13} \mathrm{C}-\mathrm{NMR}\left(75 \mathrm{MHz} \mathrm{CDCl}_{3}+\right.$ $\left.\mathrm{CD}_{3} \mathrm{OD}\right): 27.4,116.4,121.2,127.0,127.6,127.9,129.0$, 131.2, 137.0, 139.4, 158.0; EI/MS m/z (\%): $304.8\left[\mathrm{M}^{+}{ }^{\circ}\right.$; $306.8\left[\mathrm{M}^{+}\left(\mathrm{Br}^{81}, \mathrm{Cl}^{35}\right) 49\right] ; 306.8\left[\mathrm{M}^{+}\left(\mathrm{Br}^{79}, \mathrm{Cl}^{37}\right)\right.$ 17]; 308.8 $\left[\mathrm{M}^{+}\left(\mathrm{Br}^{81}, \mathrm{Cl}^{37}\right) 49\right] ;\left[\mathrm{M}^{+}-\mathrm{F}\right]=287.2 ;\left[\mathrm{M}^{+}-\mathrm{Cl}, \mathrm{F}-\right.$ fragments $]=$ 253.1; $\left[\mathrm{M}^{+}-\mathrm{CH}_{2} \mathrm{Br}\right.$, thiophene, $\mathrm{Cl}$ - fragments $]=96.1$. Anal . Calcd. For $\mathrm{C}_{11} \mathrm{H}_{7} \mathrm{BrClFS}$ : C, 43.23; H, 2.31. Found: C, 42.22; $\mathrm{H}, 2.40 \%$.

2-(bromomethyl)-5-(4-methoxyphenyl)thiophene (3b). light yellow solid,Mp: $200-250^{\circ} \mathrm{C}$; ${ }^{1} \mathrm{H}-\mathrm{NMR}(300 \mathrm{MHz}$, $\left.\mathrm{CDCl}_{3}+\mathrm{CD}_{3} \mathrm{OD}\right): \delta=7.12(\mathrm{~d}, J=8.4 \mathrm{~Hz}, 1 \mathrm{H}$-thiophene), 6.84-6.81 (m, 4H-aryl), 6.52 (d, J=3.6 Hz, 1H-thiophene), 3.98 (s, 2H-CH 2$), 2.03$ (s, 3H-OMe). ${ }^{13} \mathrm{C}-\mathrm{NMR}$ (75 MHz $\left.\mathrm{CDCl}_{3}+\mathrm{CD}_{3} \mathrm{OD}\right): 20.4,27.9,125.4,125.9,127.7,127.9$, 129.0, 129.9, 130.0, 131.9, 135.0, 139.1; EI/MS m/z (\%): $283.0\left[\mathrm{M}^{+}\right] ; 285.0\left[\mathrm{M}^{+}\left(\mathrm{Br}^{81}\right) 49\right] ;\left[\mathrm{M}^{+}-\mathrm{CH}_{2} \mathrm{Br}\right.$, thiophene $]=$ 108.2; $\left[\mathrm{M}^{+}-\mathrm{CH}_{2} \mathrm{Br}\right.$, thiophene, OMe- fragments $]=78.0$. 


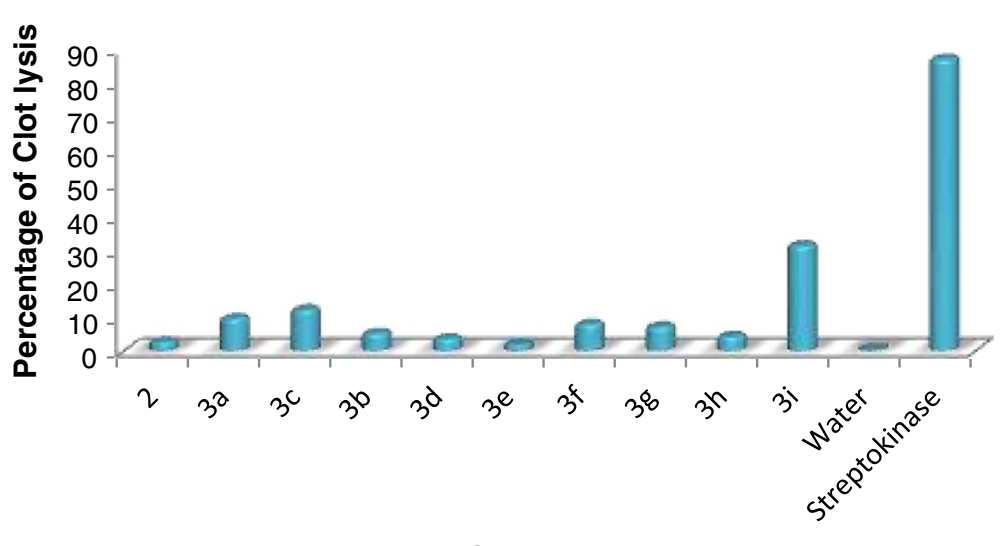

Compounds

Figure 2 Antithrombolytic activity of synthetic compounds 2, 3a-i.

Anal. Calcd. For $\mathrm{C}_{12} \mathrm{H}_{11}$ BrOS: C, 50.90; H, 3.92. Found: C, 50.76; $\mathrm{H}, 3.80 \%$.

2-(bromomethyl)-5-(4-chlorophenyl)thiophene (3c). Yellow liquid; ${ }^{1} \mathrm{H}$-NMR (300 $\mathrm{MHz}, \mathrm{CDCl}_{3}+\mathrm{CD}_{3} \mathrm{OD}$ ): $\delta=7.59$ (d, $J=8.4 \mathrm{~Hz}, 2 \mathrm{H}$-aryl), 7.40-7.37 (m, 2H-aryl, $1 \mathrm{H}$-thiophene), 7.27 (s, $1 \mathrm{H}$-thiophene), $4.70\left(\mathrm{~s}, 2 \mathrm{H}-\mathrm{CH}_{2}\right) .{ }^{13} \mathrm{C}-\mathrm{NMR}(75 \mathrm{MHz}$ $\left.\mathrm{CDCl}_{3}+\mathrm{CD}_{3} \mathrm{OD}\right): 27.6,127.5(2 \mathrm{C}), 128.5,129.5(2 \mathrm{C})$, 128.4, 131.6, 134.1, 136.7, 139.1; EI/MS (m/z -ion mode): $286.9\left[\mathrm{M}^{+}\right] ; 288.9\left[\mathrm{M}^{+}\left(\mathrm{Br}^{81}, \mathrm{Cl}^{35}\right) 49\right] ; 288.9\left[\mathrm{M}^{+}\left(\mathrm{Br}^{79}\right.\right.$, $\left.\left.\mathrm{Cl}^{37}\right) 17\right] ; 290.9\left[\mathrm{M}^{+}\left(\mathrm{Br}^{81}, \mathrm{Cl}^{37}\right) 49\right] ;\left[\mathrm{M}^{+}-\mathrm{Br}\right]=223.0$; $\left[\mathrm{M}^{+}-\mathrm{CH}_{2} \mathrm{Br}\right]=194.2 ;\left[\mathrm{M}^{+}-\mathrm{CH}_{2} \mathrm{Br}, \mathrm{Cl}\right.$ fragments $]=160.1$, $\left[\mathrm{M}^{+}-\mathrm{CH}_{2} \mathrm{Br}\right.$, Cl, aryl fragments $]=83.2$. Anal. Calcd. For $\mathrm{C}_{11} \mathrm{H}_{8}$ BrClS: $\mathrm{C}, 45.94 ; \mathrm{H}, 2.80$. Found: C, 45.90; H, $2.70 \%$.

2-(bromomethyl)-5-(3,5-difluorophenyl)thiophene (3d). Dark brown gummy matter; ${ }^{1} \mathrm{H}-\mathrm{NMR}\left(300 \mathrm{MHz}, \mathrm{CDCl}_{3}+\right.$ $\left.\mathrm{CD}_{3} \mathrm{OD}\right): \delta=7.42(\mathrm{~m}, 1 \mathrm{H}$-aryl), $7.22(\mathrm{~m}, 1 \mathrm{H}$-aryl), 7.12 (d, $J=2.4 \mathrm{~Hz}, 1 \mathrm{H}$-thiophene) $6.55(\mathrm{~m}, 1 \mathrm{H}$-aryl), 6.51 (d, $J=3.6 \mathrm{~Hz}, 1 \mathrm{H}$-thiophene), $4.51\left(\mathrm{~s}, 2 \mathrm{H}-\mathrm{CH}_{2}\right) .{ }^{13} \mathrm{C}-\mathrm{NMR}$ (75 MHz CDCl $\mathrm{CD}_{3} \mathrm{OD}$ ): 27.5, 104.0, 111.1 (2C), 127.5 (2C), 136.1, 136.9, 140.0, 165.1 (2C). EI/MS m/z (\%): 288.0 $\left[\mathrm{M}^{+}\right] ; 290.0\left[\mathrm{M}^{+}\left(\mathrm{Br}^{81}\right) 49\right] ;[\mathrm{M}-\mathrm{F}]=270.0 ;[\mathrm{M}-2 \mathrm{~F}]=254.2$; $\left[\mathrm{M}-\mathrm{CH}_{2} \mathrm{Br}\right]=196.0 ; \quad\left[\mathrm{M}-\mathrm{CH}_{2} \mathrm{Br}\right.$, thiophene fragments $]=$ 113.1. Anal. Calcd. For $\mathrm{C}_{11} \mathrm{H}_{7} \mathrm{BrF}_{2} \mathrm{~S}$ : C, 45.69; H, 2.44 . Found: C, 45.75; H, 2.43\%.

1-(3-(5-(bromomethyl)thiophene-2-yl)phenyl)ethanone (3e). Off white gummy matter; ${ }^{1} \mathrm{H}-\mathrm{NMR}\left(300 \mathrm{MHz}, \mathrm{CDCl}_{3}+\right.$ $\left.\mathrm{CD}_{3} \mathrm{OD}\right): \delta=7.87-7.85$ (m, 2H-aryl), 7.50-7.41 (m, 2Haryl), $6.91(\mathrm{~d}, J=3.6 \mathrm{~Hz}, 1 \mathrm{H}$-thiophene), $6.66(\mathrm{~d}, J=$ $3.6 \mathrm{~Hz}, 1 \mathrm{H}$-thiophene), $4.83\left(\mathrm{~s}, 2 \mathrm{H}-\mathrm{CH}_{2}\right), 2.57(\mathrm{~S}, 3 \mathrm{H}-$ COMe). ${ }^{13} \mathrm{C}-\mathrm{NMR}\left(75 \mathrm{MHz} \mathrm{CDCl}_{3}+\mathrm{CD}_{3} \mathrm{OD}\right): 26.5,27.8$, 126.0, 127.5 (2C), 128.5, 129.5, 130.5, 133.4, 136.0, 137.1, 139.0, 197.4. EI/MS m/z (\%): $294.1\left[\mathrm{M}^{+}\right] ; 296.0\left[\mathrm{M}^{+}\left(\mathrm{Br}^{81}\right)\right.$ $49] ;\left[\mathrm{M}^{+}-\mathrm{Me}\right]=281.0\left[\mathrm{M}^{+}-\mathrm{COMe}\right]=253.0,\left[\mathrm{M}^{+}-\mathrm{CH}_{2} \mathrm{Br}\right]=$ 201.1, $\left[\mathrm{M}^{+}-\mathrm{CH}_{2} \mathrm{Br}\right.$, aryl, $\left.\mathrm{COMe}\right]=84.0$. Anal. Calcd. For $\mathrm{C}_{13} \mathrm{H}_{11}$ BrOS: C, 52.89; H, 3.76. Found: C, 52.80; H, 3.66\%.

2-(bromomethyl)-5-(4-(methylthio)phenyl)thiophene (3f). off white solid,Mp: $110-150^{\circ} \mathrm{C}$; ${ }^{1} \mathrm{H}-\mathrm{NMR}(300 \mathrm{MHz}$,
$\left.\mathrm{CDCl}_{3}+\mathrm{CD}_{3} \mathrm{OD}\right): \delta=7.10(\mathrm{~m}, 4 \mathrm{H}-\mathrm{aryl}), 7.01(\mathrm{~d}, J=3.9$, $1 \mathrm{H}$-thiophene), 6.89 (d, $J=3.6,1 \mathrm{H}$-thiophene), 4.84 (s, $\left.2 \mathrm{H}-\mathrm{CH}_{2}\right) \cdot{ }^{13} \mathrm{C}-\mathrm{NMR}\left(75 \mathrm{MHz} \mathrm{CDCl}_{3}+\mathrm{CD}_{3} \mathrm{OD}\right): 14.6$, 27.8, 127.3 (2C), 127.5 (2C)127. 9 (2C), 130.0, 135.9, 139.4, 140.2. EI/MS m/z (\%): $299.0\left[\mathrm{M}^{+}\right]$; $301.0\left[\mathrm{M}^{+}\left(\mathrm{Br}^{81}\right) 49\right]$; $\left[\mathrm{M}^{+}-\mathrm{Me}\right]=284.0 ; \quad\left[\mathrm{M}^{+}-\mathrm{SMe}\right]=251.0 ; \quad\left[\mathrm{M}^{+}-\mathrm{Br}, \mathrm{Me}\right.$ fragments $]=219.0 ;\left[\mathrm{M}^{+}-\mathrm{CH}_{2} \mathrm{Br}\right.$, thiophene fragments $]=109.1$; $\left[\mathrm{M}^{+}-\mathrm{CH}_{2} \mathrm{Br}\right.$, thiophene, SMe fragments $]=77.5$. Anal. Calcd. For $\mathrm{C}_{12} \mathrm{H}_{11} \mathrm{BrS}_{2}$ : C, 48.16; H, 3.71. Found: C, 48.10; $\mathrm{H}, 3.77 \%$.

2-(bromomethyl)-5-(4-iodophenyl)thiophene (3g). Brown solid, $\mathrm{Mp}:>300^{\circ} \mathrm{C} ;{ }^{1} \mathrm{H}-\mathrm{NMR}\left(300 \mathrm{MHz}, \mathrm{CDCl}_{3}+\mathrm{CD}_{3} \mathrm{OD}\right)$ : $\delta=7.76(\mathrm{~d}, J=2.1 \mathrm{~Hz}, 2 \mathrm{H}-$ Aryl $), 7.58(\mathrm{~d}, J=1.8 \mathrm{~Hz}, 2 \mathrm{H}-$ aryl), $7.18(\mathrm{~d}, J=4.2 \mathrm{~Hz}, 1 \mathrm{H}$-Thiophene), $6.82(\mathrm{~d}, J=3.3 \mathrm{~Hz}$, $1 \mathrm{H}$-Thiophene), $4.62\left(\mathrm{~s}, 2 \mathrm{H}-\mathrm{CH}_{2}\right) .{ }^{13} \mathrm{C}-\mathrm{NMR}(75 \mathrm{MHz}$ $\left.\mathrm{CDCl}_{3}+\mathrm{CD}_{3} \mathrm{OD}\right): 27.5,94.6,127.5(2 \mathrm{C}), 129.0$ (2C), 132.0, 135.7, 138.1 (2C), 140.0. EI/MS m/z (\%): $378.1\left[\mathrm{M}^{+}{ }^{\circ}\right]$; $380.0\left[\mathrm{M}^{+}\left(\mathrm{Br}^{81}\right) 49\right] ;\left[\mathrm{M}^{+}-\mathrm{I}\right]=252.9 ;\left[\mathrm{M}^{+}\right.$-aryl, $\left.\mathrm{I}\right]=177.0$; $\left[\mathrm{M}^{+}\right.$-thiophene, aryl, $\left.\mathrm{I}\right]=95.8$. Anal. Calcd. For $\mathrm{C}_{11} \mathrm{H}_{8} \mathrm{BrIS}$ : C, 34.85; H, 2.13. Found: C, 34.80; H, 2.11\%.

2-(bromomethyl)-5-p-tolylthiophene (3h). Brown gummy matter; ${ }^{1} \mathrm{H}$-NMR $\left(300 \mathrm{MHz}, \mathrm{CDCl}_{3}+\mathrm{CD}_{3} \mathrm{OD}\right): \delta=7.55-$ 7.40 (m, 4H-aryl, 2H-thiophene), 4.68 (s, 2H), 2.35 (S, 3HMe). ${ }^{13} \mathrm{C}-\mathrm{NMR}$ (75 MHz CDCl $\left.3+\mathrm{CD}_{3} \mathrm{OD}\right): 21.0,28.1$, 125.5 (2C), 127.5 (2C), 129.6 (2C), 130.5, 131.8, 136.5, 139.6. EI/MS m/z (\%): $266.9\left[\mathrm{M}^{+}\right]$; $268.9\left[\mathrm{M}^{+}\left(\mathrm{Br}^{81}\right) 49\right]$; $\left[\mathrm{M}^{+}-\mathrm{CH}_{2} \mathrm{Br}\right]=175.0 ;\left[\mathrm{M}^{+}-\mathrm{CH}_{2} \mathrm{Br}\right.$, Me fragments $]=161.0$; $\left[\mathrm{M}^{+}-\mathrm{CH}_{2} \mathrm{Br}\right.$, thiophene fragments $]=91.0 ; \quad\left[\mathrm{M}^{+}-\mathrm{CH}_{2} \mathrm{Br}\right.$, aryl, $\mathrm{Me}$ fragments] $=83.4$. Anal. Calcd. For $\mathrm{C}_{12} \mathrm{H}_{11} \mathrm{BrS}: \mathrm{C}$, 35.94; $\mathrm{H}, 4.15$. Found: C, 35.84; $\mathrm{H}, 4.25 \%$.

2-(bromomethyl)-5-(3,5-dimethylphenyl)thiophene (3i). Brown liquid; ${ }^{1} \mathrm{H}$-NMR $\left(300 \mathrm{MHz}, \mathrm{CDCl}_{3}+\mathrm{CD}_{3} \mathrm{OD}\right): \delta=$ 7.24 (s, $1 \mathrm{H}$-aryl), 7.14 (d, $J=9.9,2 \mathrm{H}$-aryl), 7.11 (d, $J=$ $10.5 \mathrm{~Hz}, 1 \mathrm{H}$-thiophene), 6.95 (s, 1H-thiophene), 4.97 (s, $\left.2 \mathrm{H}-\mathrm{CH}_{2}\right)$; 2.35 (s, 6H-2Me). ${ }^{13} \mathrm{C}-\mathrm{NMR}\left(75 \mathrm{MHz} \mathrm{CDCl}_{3}+\right.$ $\mathrm{CD}_{3} \mathrm{OD}$ ): 21.8 (2C), 27.6, 127.4 (3C), 127.8, 130.8, 133.6, 135.9, 138.6 (2C), 139.8. EI/MS m/z (\%): $280.2\left[\mathrm{M}^{+{ }^{*}}\right]$; 
$282.2\left[\mathrm{M}^{+}\left(\mathrm{Br}^{81}\right) 49\right] ;\left[\mathrm{M}^{+}-2 \mathrm{Me}\right]=252.0 ;\left[\mathrm{M}^{+}-\mathrm{CH}_{2} \mathrm{Br}\right]=$ 189.0; $\left[\mathrm{M}^{+}-\mathrm{CH}_{2} \mathrm{Br}\right.$, aryl, $\left.2 \mathrm{Me}\right]=83.2 ;\left[\mathrm{M}^{+}-\mathrm{CH}_{2} \mathrm{Br}, \mathrm{Me}\right]^{-}=$ 174.0. Anal. Calcd. For $\mathrm{C}_{13} \mathrm{H}_{13} \mathrm{BrS}: \mathrm{C}, 55.52 ; \mathrm{H}, 4.66$. Found: C, 55.58; H, 4.63\%.

\section{Cytotoxicity studies by haemolytic assay}

The cytotoxicity of synthesized compounds 2 and $3 \mathbf{3 a - i}$ was determined by examining the haemolytic activity of human blood cells, following the previously reported method [46]. In a typical experiment, approximately $3 \mathrm{~mL}$ freshly obtained heparinized human blood was collected from the volunteers after consent and counseling. The blood samples were then centrifuged for $5 \mathrm{~min}$ at $1000 \mathrm{rpm}$, and the blood plasma was discarded and cells were washed three times with $5 \mathrm{~mL}$ chilled $\left(4^{\circ} \mathrm{C}\right)$ sterile isotonic Phosphate-buffered saline (PBS) (pH 7.4). Erythrocytes were maintained $10^{8}$ cells per $\mathrm{mL}$ for each assay. Approximately, $100 \mu \mathrm{L}$ of each synthesized compound was mixed with human blood cells $\left(10^{8}\right.$ cells $\left./ \mathrm{mL}\right)$ separately. Later on, the Samples were incubated for $35 \mathrm{~min}$ at $37^{\circ} \mathrm{C}$ and agitated after $10 \mathrm{~min}$. Soon after incubation, the samples were placed on ice for $5 \mathrm{~min}$, followed by centrifuge for $5 \mathrm{~min}$ at $100 \mathrm{rpm}$. Supernatant $100 \mu \mathrm{L}$ were taken from each tube and diluted 10 times with chilled $\left(4^{\circ} \mathrm{C}\right)$ PBS. Triton X-100 $(0.1 \% \mathrm{v} / \mathrm{v})$ was taken as positive control and phosphate buffer saline (PBS) was taken as negative control and pass through the same process. The absorbance value was measured at $576 \mathrm{~nm}$ using $\mu$ Quant (Bioteck, USA). Finally, the \% RBCs lysis for each sample was calculated.

\section{Antithrombolytic activity}

The blood samples were collected from volunteers after consent and counseling. Venous blood was pinched from healthy human volunteers without a history of anticoagulant treatment. The $100 \mu \mathrm{L}$ of blood was transmitted to each of the previously weighed micro-centrifuge tubes to form clots. Then the solution of synthesized compounds 2 and 3a-i $(100 \mu \mathrm{L})$ having concentration of $1 \mathrm{mg} / \mathrm{mL}$ was added to the tubes, and incubated at $37^{\circ} \mathrm{C}$ for 45 minutes. Streptokinase was used as standard clot lysis agent and water as negative control for this assay. Clot lysis activity results were presented in percentage [47].

\section{Conclusions}

In conclusion, novel series of 2-(bromomethyl)-5-arylthiophenes $(\mathbf{3} \mathbf{a}-\mathbf{i})$ were synthesized, and the cytotoxicity of the newly synthesized compounds $(\mathbf{2}, \mathbf{3 a - i})$ against the human blood cells was investigated. Almost, all the tested compounds revealed some haemolytic activity in the safe range but in particular $3 \mathbf{f}$ and $3 \mathbf{i}$ exhibited highest lysis of blood cells viz. 69.7 and $33.6 \%$ respectively. The synthesized compounds exhibited low to moderate antithrombolytic activity against human blood clot. The compound $\mathbf{3 i}$ was found more potent for clot lysis among all synthesized compounds. We anticipate that the continued investigation in this field will provide new insights and promote the progress towards the development of ideal thrombolytic therapy, characterized by maximized stable coronary arterial thrombolysis with minimal bleeding. The highly toxic compounds are deemed to be potential antitumor agents.

\section{Competing interests}

The authors declare that they have no competing interests.

\section{Authors' contributions}

$\mathrm{KR}, \mathrm{MZ}, \mathrm{NR}, \mathrm{MZ}, \mathrm{SA}, \mathrm{AFZ}, \mathrm{UAR}$, SUK and MS made a significant contribution to experiment design, acquisition of data, analysis and drafting of the manuscript. MZUH and HZEJ have made a substantial contribution to interpretation of data, drafting and carefully revising the manuscript for intellectual content. All authors read and approved the final manuscript.

\section{Acknowledgments}

The present data is the part of Ph.D thesis research work of Komal Rizwan. Higher Education Commission (HEC) Pakistan is highly acknowledged for financial support (Scholarschip Pin \# 2 ps1-388). The authors also gratefully acknowledge the financial support by HEC (HEC Project No. 20-1465/R\&D/ 09/5458). The authors would also like to extend their sincere appreciation to the Deanship of Scientific Research at King Saud University for funding through the Research Group Project No. RGP-VPP-345.

\section{Author details}

${ }^{1}$ Department of Chemistry, Government College University, Faisalabad 38000, Pakistan. ${ }^{2}$ Sustainable Energy Technologies (SET) Center, College of Engineering, King Saud University, PO-Box - 800, Riyadh 11421, Saudi Arabia. ${ }^{3}$ Department of Chemistry and Biochemistry, University of Agriculture, Faisalabad 38040, Pakistan. ${ }^{4}$ The Patent Office, Karachi, Pakistan. ${ }^{5}$ Department of Crop Science, Faculty of Agriculture, 43400 UPM Serdang, Selangor, Malaysia.

Received: 5 July 2014 Accepted: 27 November 2014 Published online: 17 December 2014

\section{References}

1. Chaudhary A, Jha K, Kumar S: Biological Diversity of Thiophene: A Review. J Adv Sci Res 2012, 3(3):3-10.

2. Mohan C, Bhargava G, Bedi PM: Thieno [3, 2-d] pyrimidin-4-one Derivatives as Potential Antibacterial Agents. J Life Sci 2009, 1(2):97-101.

3. Sharma S, Athar F, Maurya MR, Azam A: Copper (II) complexes with substituted thiosemicarbazones of thiophene-2-carboxaldehyde: synthesis, characterization and antiamoebic activity against E. histolytica. Eur J Med Chem 2005, 40(12):1414-1419.

4. Folkes AJ, Ahmadi K, Alderton WK, Alix S, Baker SJ, Box G, Chuckowree IS, Clarke PA, Depledge P, Eccles SA, Friedman LS, Hayes A, Hancox TC, Kugendradas A, Lensun L, Moore P, Olivero AG, Pang J, Patel S, Pergl-Wilson GH, Raynaud Fl, Robson A, Saghir N, Salphati L, Sohal S, Ultsch MH, Valenti M, Wallweber HJ, Wan NC, Wiesmann C, et al: The identification of 2-(1H-indazol-4-yl)-6-(4-methanesulfonyl-piperazin-1-ylmethyl)-4-morpholin4-yl-t hieno[3,2-d]pyrimidine (GDC-0941) as a potent, selective, orally bioavailable inhibitor of class I PI3 kinase for the treatment of cancer. J Med Chem 2008, 51(18):5522-5532.

5. Sharma C, Yerande S, Chavan R, V Bhosale A: Synthesis of thienopyrimidines and their antipsychotic activity. J Chem 2010, 7(2):655-664.

6. Abdelhamid AO: Convenient Synthesis of Some New Pyrazolo (1, 5-a pyrimidine, Pyridine, Thieno (2, 3-b pyridine, and Isoxazolo (3, 4-d pyridazine Derivatives Containing Benzofuran Moiety. J Heterocycl Chem 2009, 46(4):680-686.

7. Laddha SS, Bhatnagar SP: A new therapeutic approach in Parkinson's disease: Some novel quinazoline derivatives as dual selective phosphodiesterase 1 inhibitors and anti-inflammatory agents. Bioorg Med Chem 2009, 17(19):6796-6802. 
8. Alagarsamy V, Raja Solomon V, Meenac R, Ramaseshu K, Thirumurugan K, Murugesan S: Design and Synthesis of 2-Methylthio-3-substituted-5, 6-dimethylthieno [2, 3-d] pyrimidin-4 (3H)-ones as Analgesic, Anti-Inflammatory and Antibacterial Agents. Med Chem 2007, 3(1):67-73.

9. Wardakhan W, Abdel-Salam O, Elmegeed G: Screening for antidepressant sedative and analgesic activities of novel fused thiophene derivatives. Acta Pharm 2008, 58(1):1-14.

10. Shireesha B, UmaShankar K, RaghuramRao A, Rajan K, Raghuprasad M: Design, Synthesis and H1-Antihistaminic Activity of Novel Thieno [2, 3-d] pyrimidinones. Int J Pharm Sci Nanotechnol 2008, 1(2):136-143.

11. Connor DT, Sorenson RJ, Cetenko WA, Kerbleski JJ, Tinney FJ: Synthesis and antiallergy activity of 10-oxo-10H-pyrido [1, 2-a] thieno [3, 2-d] pyrimidines and 10-oxo-10H-pyrido [1, 2-a] thieno [3, 4-d] pyrimidines. J Med Chem 1984, 27(4):528-530.

12. Russel K, Press B, Rampulla A: Thiophene system 9, Thienopyrimidinedione derivatives as potential antihypertensive agent. J Med Chem 1988, 31:1786-1789.

13. Garnier F, Yassar A, Hajlaoui R, Horowitz G, Deloffre F, Servet B, Ries S, Alnot P: Molecular engineering of organic semiconductors: design of self-assembly properties in conjugated thiophene oligomers. J Am Chem Soc 1993, 115(19):8716-8721.

14. Garnier F, Hajlaoui R, Yassar A, Srivastava P: All-Polymer Field-Effect Transistor Realized by Printing Techniques. Science 1994, 265(5179):1684-1686.

15. Garnier F: Functionalized Conducting Polymers-Towards Intelligent Materials. Angewandte Chemie 1989, 101(4):529-533.

16. Dodabalapur A, Torsi L, Katz H: Organic transistors: two-dimensional transport and improved electrical characteristics. Science 1995, 268(5208):270-271.

17. Dodabalapur A, Katz HE, Torsi L: Molecular orbital energy level engineering in organic transistors. Adv Mater 1996, 8(10):853-855.

18. Roehrig S, Straub A, Pohlmann J, Lampe T, Pernerstorfer J, Schlemmer KH, Reinemer $P$, Perzborn E: Discovery of the novel antithrombotic agent 5chloro-N-(\{(5S)-2-oxo-3- [4-(3-oxomorpholin-4-yl)phenyl]-1,3-oxazolidin-5yl\}methyl)thiophene- 2-carboxamide (BAY 59-7939): an oral, direct factor Xa inhibitor. J Med Chem 2005, 48(19):5900-5908.

19. Dupin J, Gryglewski R, Gravier D, Hou G, Casadebaig F, Swies J, Chlopicki S: SYNTHESIS AND THROMBOLYTIC ACTIVITY. J Physiol Pharmacol 2002, 53(4):625-634

20. Miyaura N, Yanagi T, Suzuki A: The palladium-catalyzed cross-coupling reaction of phenylboronic acid with haloarenes in the presence of bases. Synthetic Comm 1981, 11(7):513-519.

21. Martin AR, Yang Y: Palladium-catalyzed cross-coupling reactions of organoboronic acids with organic electrophiles. Acta Chem Scand 1993, 47:221-230

22. Suzuki A: New synthetic transformations via organoboron compounds. Pure Appl Chem 1994, 66(2):213-222.

23. Stanforth SP: Catalytic cross-coupling reactions in biaryl synthesis. Tetrahedron 1998, 54(3-4):263-303.

24. Suzuki A: Recent advances in the cross-coupling reactions of organoboron derivatives with organic electrophiles, 1995-1998. J Organomet Chem 1999, 576(1-2):147-168.

25. Miyaura N, Suzuki A: Palladium-catalyzed cross-coupling reactions of organoboron compounds. Chem Rev 1995, 95(7):2457-2483.

26. Ali S, Rasool N, Ullah A, Nasim F-u-H, Yaqoob A, Zubair M, Rashid U, Riaz M: Design and Synthesis of Arylthiophene-2-Carbaldehydes via Suzuki-Miyaura Reactions and Their Biological Evaluation. Molecules 2013, 18(12):14711-14725.

27. Dang TT, Rasool N, Dang T, Reinke H, Langer P: Synthesis of tetraarylthiophenes by regioselective Suzuki cross-coupling reactions of tetrabromothiophene. Tetrahedron Lett 2007, 48(5):845-847.

28. Langle S, Abarbri M, Duchêne A: Selective double Suzuki cross-coupling reactions. Synthesis of unsymmetrical diaryl (or heteroaryl) methanes. Tetrahedron Lett 2003, 44(52):9255-9258.

29. Bandgar B, Bettigeri SV, Phopase J: Palladium catalyzed ligand-free Suzuki cross-coupling reactions of benzylic halides with aryl boronic acids under mild conditions. Tetrahedron Lett 2004, 45(37):6959-6962.

30. Molander GA, Elia MD: Suzuki-Miyaura cross-coupling reactions of benzyl halides with potassium aryltrifluoroborates. J Org Chem 2006, 71(24):9198-9202.

31. McDaniel SW, Keyari CM, Rider KC, Natale NR, Diaz P: Suzuki-Miyaura Cross-Coupling of Benzylic Bromides Under Microwave Conditions. Tetrahedron Lett 2011, 52(43):5656-5658.
32. Dittmer K, Martin RP, Herz W, Cristol SJ: The Effect of Benzoyl Peroxide on the Bromination of Methylthiophenes by N-Bromosuccinimide. J Am Chem Soc 1949, 71(4):1201-1204.

33. Negishi E-i, de Meijere A, Wiley J: Handbook of organopalladium chemistry for organic synthesis, Volume 1. New York: Wiley-Interscience; 2002

34. Juteau H, Gareau Y, Labelle M, Sturino CF, Sawyer N, Tremblay N, Lamontagne S, Carriere MC, Denis D, Metters KM: Structure-activity relationship of cinnamic acylsulfonamide analogues on the human EP3 prostanoid receptor. Bioorg Med Chem 2001, 9(8):1977-1984.

35. Smith GB, Dezeny GC, Hughes DL, King AO, Verhoeven TR: Mechanistic studies of the Suzuki cross-coupling reaction. J Org Chem 1994, 59(26):8151-8156.

36. Tùng ĐT, Tuân ĐT, Rasool N, Villinger $A$, Reinke $H$, Fischer $C$, Langer $P$ : Regioselective Palladium (0)-Catalyzed Cross-Coupling Reactions and Metal-Halide Exchange Reactions of Tetrabromothiophene: Optimization, Scope and Limitations. Adv Synth Catal 2009, 351(10):1595-1609.

37. Mavrova A, Wesselinova D, Tsenov YA, Denkova P: Synthesis, cytotoxicity and effects of some 1,2,4-triazole and 1,3,4-thiadiazole derivatives on immunocompetent cells. Eur J Med Chem 2009, 44(1):63-69.

38. Mologni L, Rostagno R, Brussolo S, Knowles PP, Kjaer S, Murray-Rust J, Rosso E, Zambon A, Scapozza L, McDonald NQ: Synthesis, structure-activity relationship and crystallographic studies of 3-substituted indolin-2-one RET inhibitors. Bioorg Med Chem 2010, 18(4):1482-1496.

39. Ding $H$, Chen $Z$, Zhang $C$, Xin $T$, Wang $Y$, Song $H$, Jiang $Y$, Chen $Y, X u Y$, Tan C: Synthesis and Cytotoxic Activity of Some Novel N-Pyridinyl-2(6-phenylimidazo[2,1-b]thiazol-3-yl)acetamide Derivatives. Molecules 2012, 17(4):4703-4716.

40. Panak E, Maffrand J, Picard-Fraire C, Vallee E, Blanchard J, Roncucci R: Ticlopidine: a promise for the prevention and treatment of thrombosis and its complications. Haemostasis 1983, 13(Suppl 1):1-54

41. Coukell AJ, Markham A: Clopidogrel. Drugs 1997, 54(5):745-750. discussion 751

42. Mucklow J: Thrombolytic treatment. Streptokinase is more economical than alteplase. BMJ 1995, 311(7018):1506.

43. Collen D: Coronary thrombolysis: streptokinase or recombinant tissue-type plasminogen activator? Ann Intern Med 1990, 112(7):529-538.

44. Hunt BJ: Awareness and politics of venous thromboembolism in the United kingdom. Arterioscler Thromb Vasc Biol 2008, 28(3):398-399.

45. Allroggen $\mathrm{H}$, Abbott RJ: Cerebral venous sinus thrombosis. Postgrad Med J 2000, 76(891):12-15.

46. Shahid M, Bukhari SA, Gul Y, Munir H, Anjum F, Zuber M, Jamil T, Zia KM: Graft polymerization of guar gum with acryl amide irradiated by microwaves for colonic drug delivery. Int J Biol Macromol 2013, 62:172-179.

47. Prasad S, Kashyap RS, Deopujari JY, Purohit HJ, Taori GM, Daginawala HF: Development of an in vitro model to study clot lysis activity of thrombolytic drugs. Thromb J 2006, 4(14):1-4.

Publish with ChemistryCentral and every
scientist can read your work free of charge
"Open access provides opportunities to our
colleagues in other parts of the globe, by allowing
anyone to view the content free of charge."
W. Jeffery Hurst, The Hershey Company.
- available free of charge to the entire scientific community
- peer reviewed and published immediately upon acceptance
- cited in PubMed and archived on PubMed Central
- yours - you keep the copyright
submit your manuscript here:
http://www.chemistrycentral.com/manuscript/

